\title{
Spectroscopic and Structural Insight into the Size-Dependent Behavior of the Nanophase
}

\author{
Santanu Bera, ${ }^{1}$ Sandip Dhara, ${ }^{2}$ Alexander Milekhin, ${ }^{3}$ M. W. Chu, ${ }^{4}$ and S. M. Shivaprasad ${ }^{5}$ \\ ${ }^{1}$ Water and Steam Chemistry Division, Bhabha Atomic Research Centre Facilities, Kalpakkam 603102, India \\ ${ }^{2}$ Surface and Nanoscience Division, Indira Gandhi Centre for Atomic Research, Kalpakkam 603102, India \\ ${ }^{3}$ Institute of Semiconductor Physics, Russian Academy of Sciences, 630090 Novosibirsk, Russia \\ ${ }^{4}$ Center for Condensed Matter Sciences, National Taiwan University, Taipei 106, Taiwan \\ ${ }^{5}$ Jawaharlal Nehru Centre for Advanced Scientific Research, International Centre for Materials Science and Chemistry, \\ Bangalore 560064, India
}

Correspondence should be addressed to Santanu Bera, bera@igcar.gov.in

Received 27 October 2011; Accepted 27 October 2011

Copyright (C) 2012 Santanu Bera et al. This is an open access article distributed under the Creative Commons Attribution License, which permits unrestricted use, distribution, and reproduction in any medium, provided the original work is properly cited.

Nanoscience and nanotechnology demand high-quality spectroscopic characterization and an in-depth understanding of spectral characteristics of nanomaterials. Several methodologies combining microscopy and spectroscopy are adapted to explain the special characteristics of the nanomaterials. In this special issue, it is aimed to bring the recent research papers which explain the special features in spectroscopic data and its analyses in case of nanostructures. Here, we have selected six papers, limited to few spectroscopic techniques. This is of course not the state of the art of total spectroscopic studies in this field. The selected papers in this topic are merely representing the type and trend of work in this area and are not really depicting a complete picture. We thank the authors for their excellent contributions to this issue. The critical reviews and comments by the reviewers are very helpful in improving some of the papers, and their contributions are gratefully acknowledged.

This special issue contains six papers, where five papers deal with vibrational spectroscopy and one paper deals with the electron spectroscopy of nanostructured materials.

The first paper entitled "Phonon spectra of small colloidal II-VI semiconductor nanocrystals" by V. Dzhagan et al. explores the first- and higher-order phonon spectra of several kinds of II-VI nanocrystals using resonant Raman spectroscopy to have a unified view onto the vibrational spectrum of semiconductor nanocrystals. The combinational modes consisting either of longitudinal and transverse or surface optical modes is accounted for the lineshape of the spectrum of the first-order modes. The paper shows that the Electronphonon coupling-mediated strong Fröhlich interaction in II-VI compounds prohibits observation of second-order transverse and surface optical modes. A broad surface optical mode is found to dominate the resonant phonon Raman spectrum of nanocrystals smaller than $2 \mathrm{~nm}$.

The second paper entitled "Size-dependent non-fret photoluminescence quenching in nanocomposites based on semiconductor quantum dots CdSe/ZnS and functionalized porphyrin ligands" by E. I. Zenkevich et al. shows strong quenching of quantum dots (QDs) photoluminescence (PL) in "QD-Porphyrin" nanocomposites based on colloidal CdSe/ZnS and CdSe QDs surface modified with tetra-mesopyridyl substituted porphyrin molecules, and its possible origin is reported from steady-state and picosecond timeresolved measurements. QD PL quenching rate constants are found inversely proportional to QD diameter. The comparative study of experimental data and quantum mechanical calculations lead to the inference of QD PL quenching as an effect of tunneling of the electron of the excited electronhole pair followed by a self-localization of the electron or formation of trap states. The calculated quantum-confined exciton wave function at the QD surface is thought to be the major contributing factor to PL quenching. It has been highlighted in the study that a single functionalized molecule is good enough to be one of the probes for the complex interface physics and dynamics of colloidal semiconductor QDs. 
The third paper entitled "Analysis on binding energy and Auger parameter for estimating size and stoichiometry of $\mathrm{ZnO}$ nanorods" by S. Bera et al. relates the features of photoelectron spectra with the nanosize tips of $\mathrm{ZnO}$ nanorods. The SEM picture of the nanorods shows the rod ends composed of nanosize tips which significantly change the binding energy of Zn 2p photoelectron peaks. The paper explains the shift in binding energy in terms of the change in relaxation energy of the photoelectrons coming out of the nanotips of the oxide.

The fourth paper entitled "Raman spectra of quaternary $C d S_{1-x-y} S_{x} T e_{y}$ nanocrystals embedded in borosilicate glass" by Azhniuk et al. reports about a set of samples with quaternary $\mathrm{CdS}_{1-x-y} \mathrm{Se}_{x} \mathrm{Te}_{y}$ nanocrystals grown in a borosilicate glass matrix by the diffusion-limited growth technique with three types of cations distributed over nanocrystal lattice sites. The Raman spectra confirm the three-mode compositional behavior of phonon modes in these crystals. The compositional dependence of the longitudinal optical phonon frequencies has been studied for a set of nanocrystalline samples having composition with nearly equal stoichiometry of Se and Te.

The fifth paper entitled "Vibrational spectroscopy of chemical species in silicon and silicon-rich nitride thin films" by K. O. Bugaev et al. reports vibrational properties of hydrogenated silicon-rich nitride of various stoichiometry and hydrogenated amorphous silicon (a-Si:H) films using Raman and Fourier transform infrared (FTIR) spectroscopic techniques. FTIR analyses show formation of Si$\mathrm{H}$ bonds in the thermally and laser-annealed samples of partial crystallized silicon nitride with near stoichiometric composition. On the other hand, Raman spectroscopic analyses show presence of $\mathrm{Si}-\mathrm{H}$ bonds for a-Si:H films with hydrogen concentration $\leq 15 \%$ and existence of $\mathrm{Si}-\mathrm{H}_{2}$ bonds for hydrogen concentration of 30-35\%. Nanosecond-pulsed laser treatments show crystallization of these films and its dehydrogenization.

The sixth paper entitled "Room temperature synthesis and catalytic properties of surfactant-modified Ag nanoparticles" by $\mathrm{W}$. Li et al. mostly concentrates on the preparation of uniform size nano-Ag particle through wet chemistry route at room temperature. Regular spectral features observed in FTIR and UV absorption spectra along with TEM analyses have been coordinated to understand the character of the nano particles. Suspended nano-Ag of size $20-30 \mathrm{~nm}$ showed extremely good catalytic property. However, less importance is given in correlating the spectral features observed in FTIR or UV absorption spectra with the size of the nanoparticle, and this is mostly because the sizes are of the order of 20 $30 \mathrm{~nm}$.

\author{
Santanu Bera \\ Sandip Dhara \\ Alexander Milekhin \\ M. W. Chu \\ S. M. Shivaprasad
}




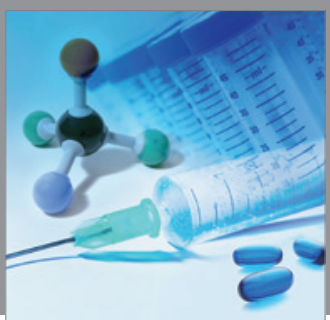

International Journal of

Medicinal Chemistry

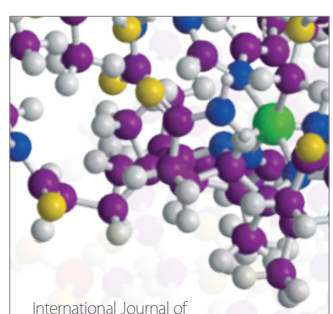

Carbohydrate Chemistry

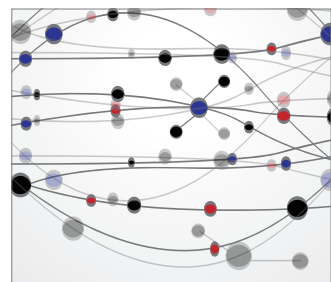

The Scientific World Journal
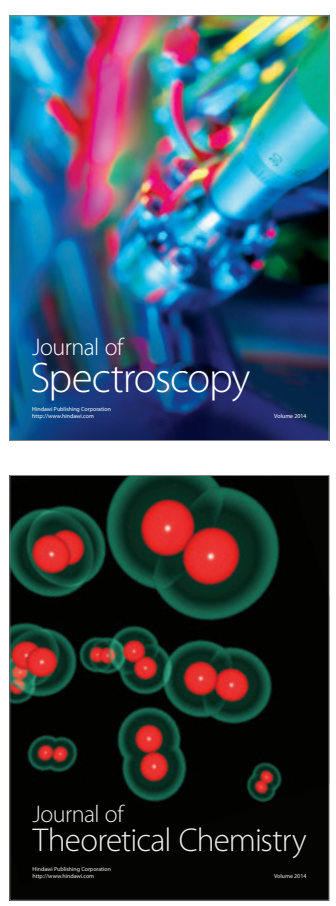
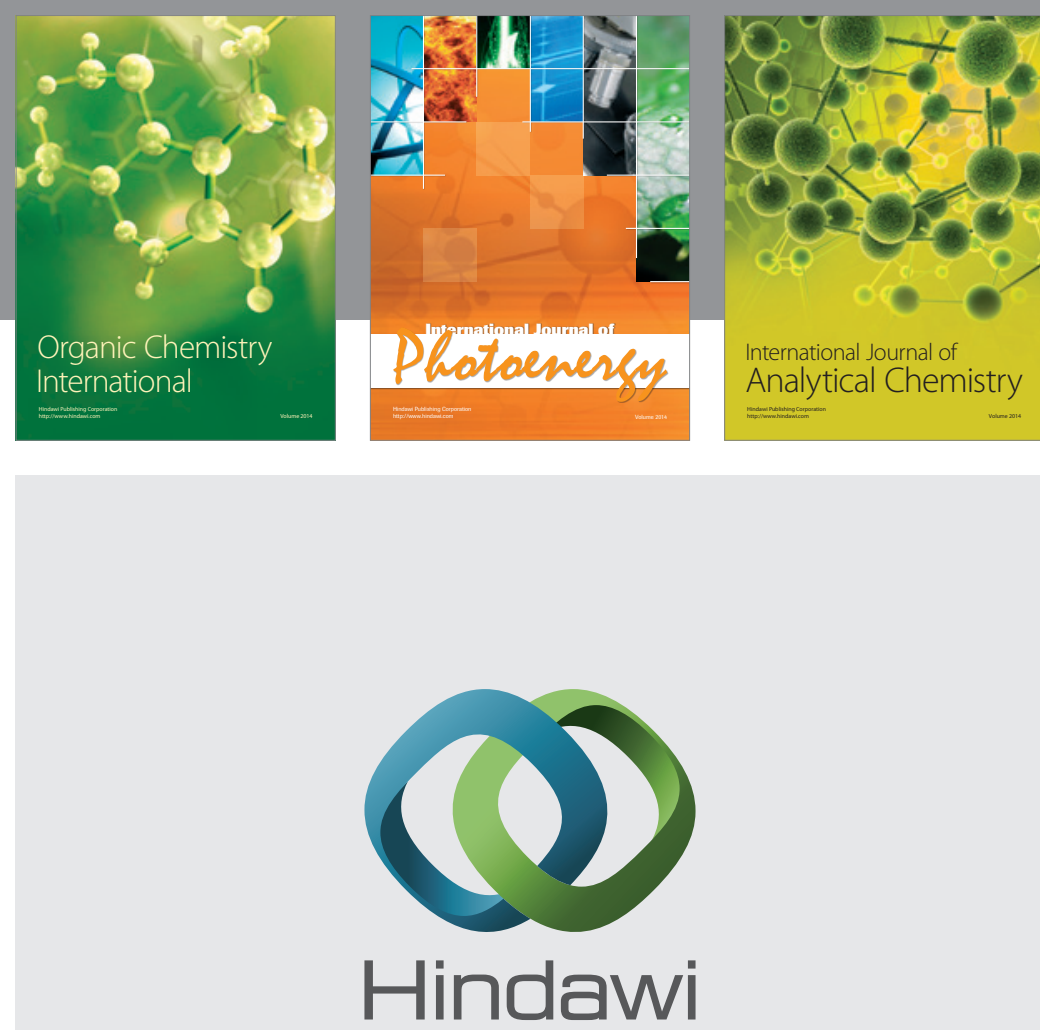

Submit your manuscripts at

http://www.hindawi.com
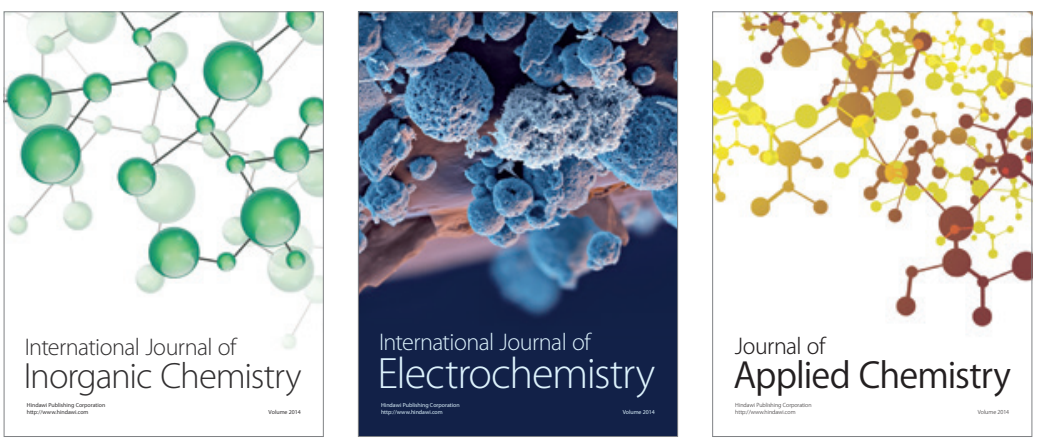

Journal of

Applied Chemistry
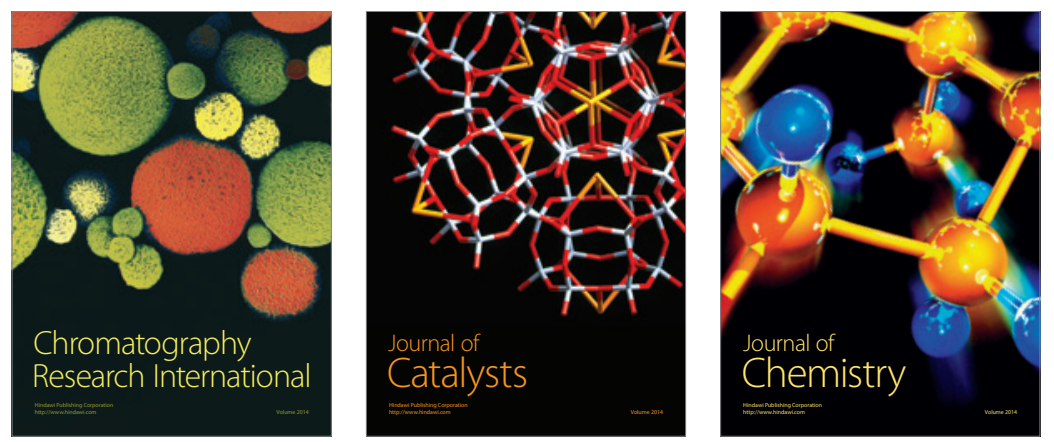
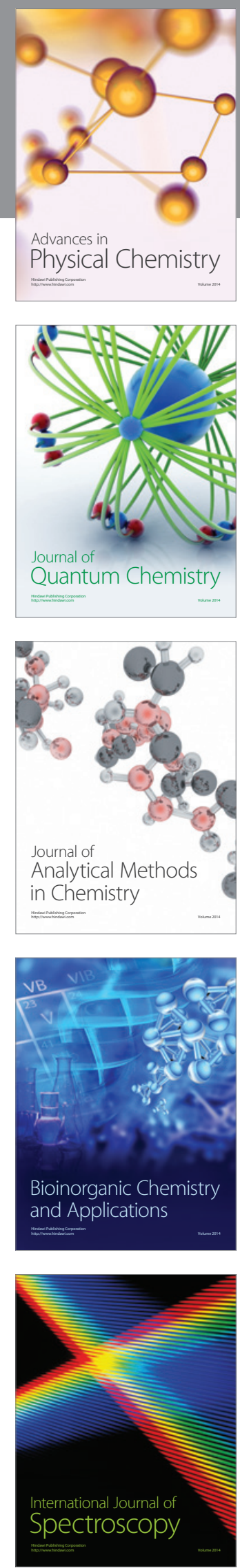